\title{
Diagnóstico participativo de calidad de agua como herramienta para la gestión ambiental. El caso del Municipio de Vichadero (Rivera, Uruguay)
}

\author{
PARTICIPATORY DIAGNOSIS OF WATER QUALITY AS A TOOL FOR \\ ENVIRONMENTAL MANAGEMENT. THE CASE OF THE MUNICIPALITY OF \\ VICHADERO, RIVERA, URUGUAY \\ Mosqueira Campos, A.* Lavaggi, M.L.*1 \\ amosqueira@fcien.edu.uy \\ maria.lavaggi@cur.edu.uy \\ Recibido: 30/09/2019 Aceptado: 20/12/2019
}

\begin{abstract}
Resumen
La presente investigación surge a partir de preocupaciones manifestadas por actores sociales locales del municipio de Vichadero, Rivera, Uruguay y de la inexistencia de datos sobre las condiciones que presentan los cuerpos de agua de la zona. El objetivo fue identificar las principales problemáticas ambientales de la zona en base a la percepción de la comunidad, a partir de un diagnóstico socio-ambiental utilizando metodologías participativas y empoderar a través de la producción colectiva del conocimiento a las personas involucradas. Este abordaje se puso en diálogo con resultados de análisis físicoquímicos de la calidad del agua superficial en las microcuencas de la zona. Esta investigación da evidencia de que procesos similares podrían promover el involucramiento de actores locales en procesos de monitoreo e investigación asociados a la gestión ambiental de una localidad.
\end{abstract}

Palabras clave: calidad de agua; diagnóstico participativo; percepción ambiental

\begin{abstract}
The current investigation comes from concerns expressed by local social actors in the municipality of Vichadero, Rivera, Uruguay and the lack of data on the conditions of the water bodies in the area. The objective was to identify the main environmental problems of the area based on a socio-environmental diagnosis using participatory methodologies and empowering the collective production of knowledge. This approach was put in dialogue with the results of physical-chemical analysis of the quality of surface water in the micro watershed of the area. This research provides evidence that similar processes could promote the involvement of local actors in monitoring and research processes associated with the environmental management of a locality.
\end{abstract}

Keywords: water quality; participatory diagnosis; environmental perception

1 *_Centro Universitario Regional Noreste, sede Rivera-Universidad de la República

Tekoporá ${ }^{\circledR}$. Centro Universitario de la Región Este. Universidad de la República

(C) Mosqueira y Lavaggi (2020)

Este es un artículo de Acceso Abierto distribuido bajo licencia Creative Commons (CC BY NC 4.0) 


\section{Introducción}

La buena calidad del agua depende de su gestión sustentable, integrada y participativa. Para lograr una gestión integrada de los recursos hídricos se requiere que todos los usos del agua se consideren en conjunto, debido a que todas las actividades que se desarrollan en el territorio los afectan (Aguirre, 2011; Ministerio de Vivienda Ordenamiento Territorial y Medio Ambiente [MVOTMA], 2017). Esta concepción de gestión integrada no solo requiere conciencia colectiva, compromiso y capacitación, sino además discusiones amplias y participativas en los procesos de toma de decisiones. Esto implica el desarrollo de la capacidad de las comunidades y de todas y todos los actores sociales, a fin de la comprensión general de los procesos. Consecuentemente les permitirá convertirse en actores fundamentales para detener acciones destructivas y promover acciones de conservación y restauración (Grosse, Santos, Taks y Thimel, 2006).

El territorio tiene un vínculo directo con las dinámicas de las aguas superficiales y los factores que la regulan. El concepto de ambiente en este contexto no es un objeto ni una relación en el sentido sistémico ya que no separa la sociedad y la naturaleza del ambiente, sino que lo considera como forma compleja e integrada, surge la necesidad de construir un abordaje continuo, racional y ambiental, diferente a un abordaje urgente, más volcado a lo económico e instrumental, por ende el ambiente se sitúa desde una crítica al mundo actual (Eschenhagen, 2007).

Las unidades espaciales definidas en el proceso de circulación de las aguas superficiales se denominan cuencas hidrográficas (Achkar, Díaz, Domínguez y Pesce, 2016). Una cuenca es un sistema compuesto por una gran diversidad de componentes bióticos y abióticos que interactúan entre sí, por lo cual cualquier alteración que ocurra en este sistema complejo tendrá incidencia en el funcionamiento global, como el de sus subsistemas componentes, las microcuencas (Achkar, Domínguez y Pesce, 2004). La introducción de agentes extraños al sistema, ya sean de origen orgánico o inorgánico, puede afectarlo de forma negativa o positiva. Una evaluación sistemática, un monitoreo periódico y estandarizado de la calidad del agua, es de vital importancia para tener conocimiento de las posibles variaciones de los parámetros que pudieran comprometer la calidad del recurso hídrico. 
El aprovechamiento integral de los bienes ambientales contenidos en las distintas cuencas hidrográficas, impulsan la gestión ambientalmente sustentable del territorio. Esta gestión tiene como finalidad el mejoramiento de las condiciones de vida de la comunidad local y de la calidad ambiental. La gestión ambientalmente sustentable combina en forma sinérgica los saberes científicos y técnicos con los saberes de las comunidades locales, considerando en esta combinación las percepciones ambientales locales. De esta forma se impulsa el compromiso y la participación real de las y los actores directamente involucrados con el manejo de los recursos naturales (Achkar et al., 2004).

El conocimiento de la percepciones ambientales nos da lugar a conocer el porqué de las acciones, las prácticas, las interpretaciones y los significados que dan fundamento a la elaboración de juicios en relación con las sensaciones obtenidas del ambiente (Vargas, 1994). De esta forma, los estudios sobre percepciones ambientales son considerados mecanismos de aproximación de la comunidad, favoreciendo la participación, análisis y comunicación de impactos ambientales en sus territorios (Abreu, Sabóia, Nascimento y de Souza, 2017). Según Chaui (1996), la percepción es una comunicación entre nuestro cuerpo, el cuerpo de los otros sujetos y los cuerpos de las cosas. Tratándose de una comprensión holística ser humanoambiente, donde las personas son moldeadas por las condiciones ambientales donde viven (Benez, Kauffer Michel y Álvarez Gordillo, 2010). Cabe destacar que en Uruguay son escasas las investigaciones sobre recursos hídricos y su gestión, que consideren la percepción ambiental como fuente de información.

Dentro de las posibles metodologías para abordar las percepciones ambientales, podemos distinguir entre aquellas que involucran en forma activa a la comunidad y aquellas en la que la información proveniente de la comunidad es analizada sin la participación de los mismos (Fernández, 2008). Una de las formas de abordar las percepciones ambientales con participación activa de la comunidad es la Investigación Acción Participativa (IAP). Esta estrategia de investigación enfatiza la participación comunitaria y la construcción colectiva como conceptos primordiales y toma como base los intereses y preocupaciones locales. Metodología que se orienta hacia la transformación social con carácter crítico, dialógico, reflexivo, político, fortalecedor de la sociedad civil y democrático (Sevilla Guzmán, 2001). 
La IAP no acepta el pensamiento único y excluyente, a la vez que intenta generar nuevos conocimientos a partir de la integración y la puesta en diálogo de varios saberes. A través de la IAP no se busca solamente conocer y explicar, se trata de intervenir y articularse con las problemáticas que se plantea desde el grupo de trabajo. Una vez situada la IAP será posible conseguir el salto de acción social colectiva a acción de movimiento social. Al presentar un carácter participativo la IAP debe tener autenticidad y compromiso, donde las y los participantes sean agentes de cambio y no víctimas de una situación (Fals Borda, 1981; Balcazar, 2003).

El diagnóstico socio-ambiental de un territorio debe ser desarrollado en base a estudios de percepción comunitaria como una estrategia de aproximación de la comunidad, dando a conocer sus prácticas, valores y costumbres, así como también su participación en la identificación de problemas ambientales (Abreu et al., 2017). Como plantea Abreu et al., (2017), la IAP como una estrategia que aborda la percepción ambiental, es un importante instrumento para la planificación de acciones y políticas, ya que una de las dificultades para lograr una real conservación de los recursos naturales es el no involucramiento de actores locales en los espacios de toma de decisiones-

El Estado uruguayo como uno de los actores que cumple el rol de promover acciones de conservación y restauración de los recursos naturales, presenta normativas que incluyen la participación ciudadana en la gestión ambiental, incluyendo la Constitución de la República y normativas ambientales como la Ley General de Protección Ambiental ( $N^{\circ}$ 17.283), la Ley que crea el Sistema Nacional de Áreas Protegida ( $\mathrm{N}^{\circ}$ 17.234), la Ley de Evaluación de Impacto Ambiental $\left(\mathrm{N}^{\circ}\right.$ 16.466), la Ley de Ordenamiento Territorial y Desarrollo Sostenible $\left(\mathrm{N}^{\circ}\right.$ 18.308), la Política Nacional de aguas ( $N^{\circ}$ 18.610) y el Plan Nacional de Aguas aprobado por decreto del Poder Ejecutivo en el año 2017. A nivel departamental, existe la ordenanza de Ordenamiento Territorial del Departamento de Rivera (№ 03/2007). La gestión integrada de los recursos hídricos es una práctica que los ciudadanos y las ciudadanas aún encuentran lejana, más allá del marco institucional descrito anteriormente, que reconoce en la participación ciudadana uno de sus pilares (Ríos, 2012).

Por otro lado en Uruguay la eutrofización de los ríos y arroyos se debe principalmente a los cambios de uso de suelo que se han realizado en las cuencas hidrográficas, provocando 
problemáticas en sus funciones ecosistémicas y en los servicios que brinda. Esta degradación de la calidad de agua ocurre debido al aporte de materia orgánica y nutrientes inorgánicos (detergentes, fertilizantes), en algunas ocasiones ésta puede ser de carácter puntual (origen urbano e industrial) o difuso (origen agropecuario).

La calidad de agua de un curso es determinada en función de rangos de diferentes parámetros que determinan su uso potencial (De León, 2011). En Uruguay según el decreto reglamentario 253/979 (Ley 14.859, Uruguay) que hace referencia a la normativa en materia de calidad de agua, todo cuerpo de agua continental que presente una cuenca tributaria mayor a $10 \mathrm{~km}^{2}$, quedará comprendido dentro de la clase 3 del decreto reglamentario. Este decreto representa la referencia de calidad de agua a nivel nacional y establece valores límites para determinados parámetros que se usan para su determinación.

El presente trabajo surge a partir de la participación de una de las docentes del laboratorio en Química Ambiental del Centro Universitario de Rivera (CUR) en espacios de educación formal de Vichadero. En reiteradas ocasiones, algunos actores sociales de la comunidad mencionan la preocupación sobre los posibles riesgos a los que están expuestos, por causa de la contaminación ambiental asociada a la actividad antropogénica en la zona. Es a partir de estas preocupaciones manifestadas y de la inexistencia de datos sobre las condiciones que presentan los cuerpos de agua de la localidad y sus zonas aledañas, que surge el proyecto del presente trabajo.

El objetivo de la presente investigación fue promover metodologías participativas para realizar un diagnóstico socio-ambiental sobre la calidad del agua superficial en las microcuencas ubicadas en la zona del municipio de Vichadero. Este abordaje se contrastó con resultados de análisis físico-químicos de la calidad del agua en la zona realizados en la zona. Esto permitió determinar cuáles factores se encuentran influenciando esta percepción y empoderar a través de la producción colectiva del conocimiento a las y los actores sociales inmersos en sus propias problemáticas.

\section{Metodologías aplicadas durante la investigación}

Durante el desarrollo de esta investigación se combinaron metodologías complementarias según las diferentes etapas. Se caracterizó la zona de estudio y se utilizaron 
los Sistemas de Información Geográfica (SIG) como una herramienta fundamental para el análisis y muestreo (Fernández, 2005). El SIG se desarrolló utilizando el Software ArcGis 10.1 (Environmental Systems Research Institute [ESRI], 2012). Se realizó un enfoque en el proceso participativo, a partir de la convocatoria, posterior identificación de posibles conflictos ambientales y diseño de muestreo en conjunto con el grupo de referencia, a lo que llamamos mapeo colectivo (Reisler y Ares, 2013).

En base al tipo de información relevada y las capacidades locales instaladas de análisis, se seleccionaron los siguientes parámetros de calidad de agua: temperatura, $\mathrm{pH}$, conductividad, sólidos disueltos totales, oxígeno disuelto, concentración de Nitratos (Brandao, 2014; Instituto de Hidrología, Meteorología y Estudios Ambientales [IDEAM], 2007), fósforo y demanda bioquímica de oxígeno (Dirección Nacional de Medio Ambiente [DINAMA], 2009a). Estos parámetros brindan información sobre el estado de los cuerpos de agua y posibles procesos de eutrofización.

\section{Área de estudio}

El área de estudio corresponde a las microcuencas del arroyo Coronilla y del arroyo Coronilla Chico, ambas comprendidas en la cuenca alta del Río Negro, en el sureste del departamento de Rivera, Uruguay (Figura 1). La microcuenca del arroyo Coronilla representa una superficie de $183 \mathrm{~km}^{2}$ y la microcuenca arroyo Coronilla Chico una superficie de $75 \mathrm{~km}^{2}$, ambas pertenecen a la ecorregión denominada colinas del noreste ganaderas y agrícolas. Presenta diversidad de suelos, desde superficies con afloramientos hasta suelos profundos con fertilidad variable (Achkar et al., 2016). La región es tradicionalmente ganadera extensiva, se practica agricultura, y en ambas microcuencas predominan los usos de suelo herbáceo natural y campos de cultivos, principalmente de arroz y de soja (Dirección Nacional de Ordenamiento Territorial [DINOT], 2015) (Figura 2). Además, en el área de estudio fueron identificadas zonas con presencia de ecosistemas degradados y zonas con importante utilización de agroquímicos (Achkar et al., 2004). Existen en la zona predios cercanos al municipio donde se cultivan arroz, soja y otros cultivos, superan las 5000 ha, estas actividades están siendo impulsadas por capitales extranjeros provenientes de Brasil y Argentina (Programa de las Naciones Unidas para el medio ambiente [PNUMA], 2009). 
El municipio de Vichadero se encuentra dentro del área de estudio, ubicado en empalme de ruta 6 y 27, muy cerca de la frontera con Brasil, a 96kms de la ciudad de Bagé. Vichadero fue declarado municipio en el año 2010 y presenta una superficie de 510,5 km². El municipio cuenta con una población aproximadamente de 4048 habitantes, según el censo realizado por el Instituto Nacional Estadístico (INE) del año 2011. Esta localidad no posee una red de saneamiento generalizado, existiendo viviendas que vierten las aguas residuales, domésticas principalmente, a las cunetas de la vía pública; pozos negros y servicio de extracción con barométrica, o infiltración a terreno en forma más o menos directa. Los envases de productos fitosanitarios utilizados en agricultura de arroz y soja y en sanidad animal, no tienen destino final fijo y son empleados por la población para diversos fines domésticos, sin un adecuado tratamiento posterior a su uso.

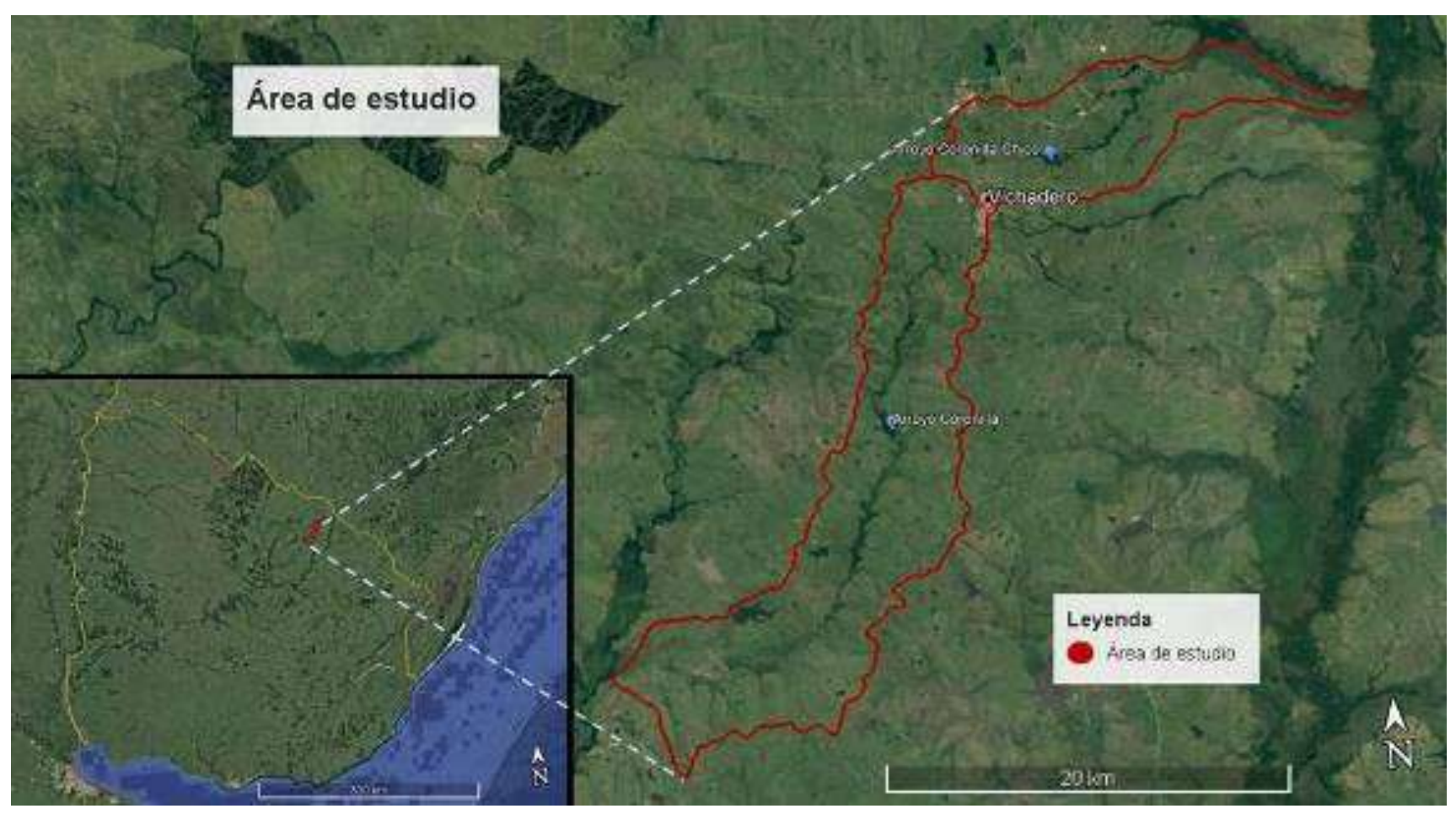

Figura 1. Área de estudio (Fuente: Google Earth Pro, imagen 2018) 

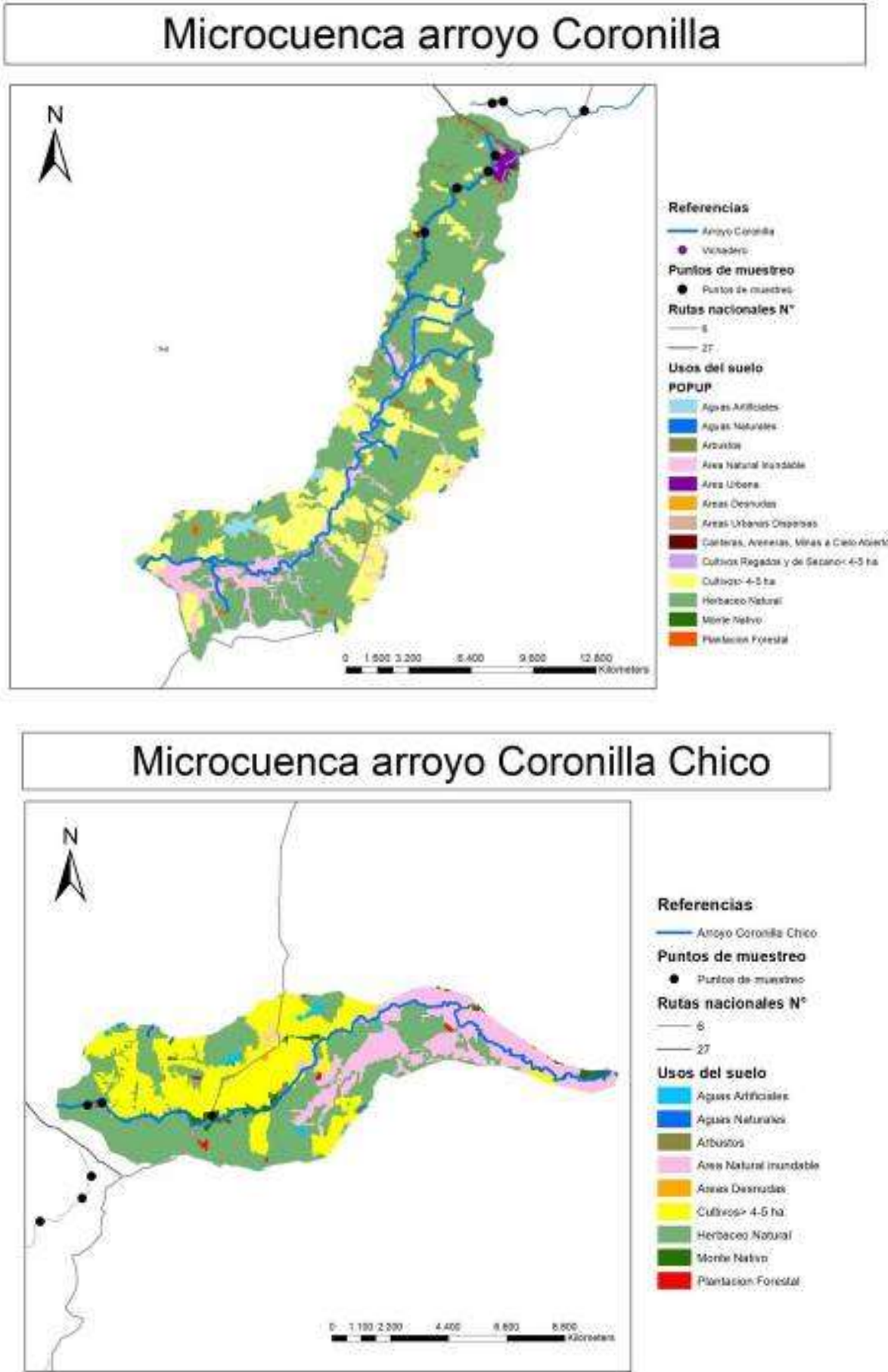

Figura

2.

Microcuenca arroyos

Coronilla y Coronilla Chico, puntos de muestreo y usos de suelo.

(Fuente: elaboración propia).

\section{Proceso}

\section{Participativo}

El diagnóstico participativo se desarrolló entre julio 2017 y noviembre 2018., En él participaron actores sociales locales del municipio de Vichadero y se realizó un diagnóstico sobre la calidad de agua de la zona. En conjunto con los actores locales que participaron se identificaron las problemáticas

ambientales de la zona, se realizó el diseño de muestreo, la toma de muestras de agua y se discutieron los resultados.

Las herramientas participativas fueron utilizadas en las diferentes etapas del proyecto, tanto en la fase de presentación de la propuesta, como en la fase de análisis y evaluación del problema para asegurar que el proceso esté accesible a todas las personas involucradas e 
interesadas (Geilfus, 2002). En el marco de los objetivos, este proyecto se enfocó en el diagnóstico participativo socio-ambiental, como estrategia de concientización y movilización. El diagnóstico participativo ambiental es un proceso que introduce a la auto reflexión crítica, su finalidad es la visualización y transformación de las relaciones sociedad-ambiente y dar respuesta a determinados problemas generados por éstas, partiendo de la acción-reflexión de las y los integrantes de la comunidad (Abreu et al., 2017).

Este proceso puede ser dividido en tres instancias complementarias: taller, campo y laboratorio, sistematizadas abajo a través del Cuadro 1. Las primeras instancias se realizaron en formato taller, con la finalidad de poder intercambiar con profundidad acerca de qué se espera lograr, la temática a ser abordada y sobre qué territorios. Las instancias a campo complementan las instancias en formato taller, permitiendo incorporar dimensiones temporales, corporales al proceso. Se realizan registros y recolección de insumos visuales y sensoriales para elaborar relatos. Las instancias en laboratorio acercan a los actores sociales a un saber específico técnico, además de introducirlos a la institución.

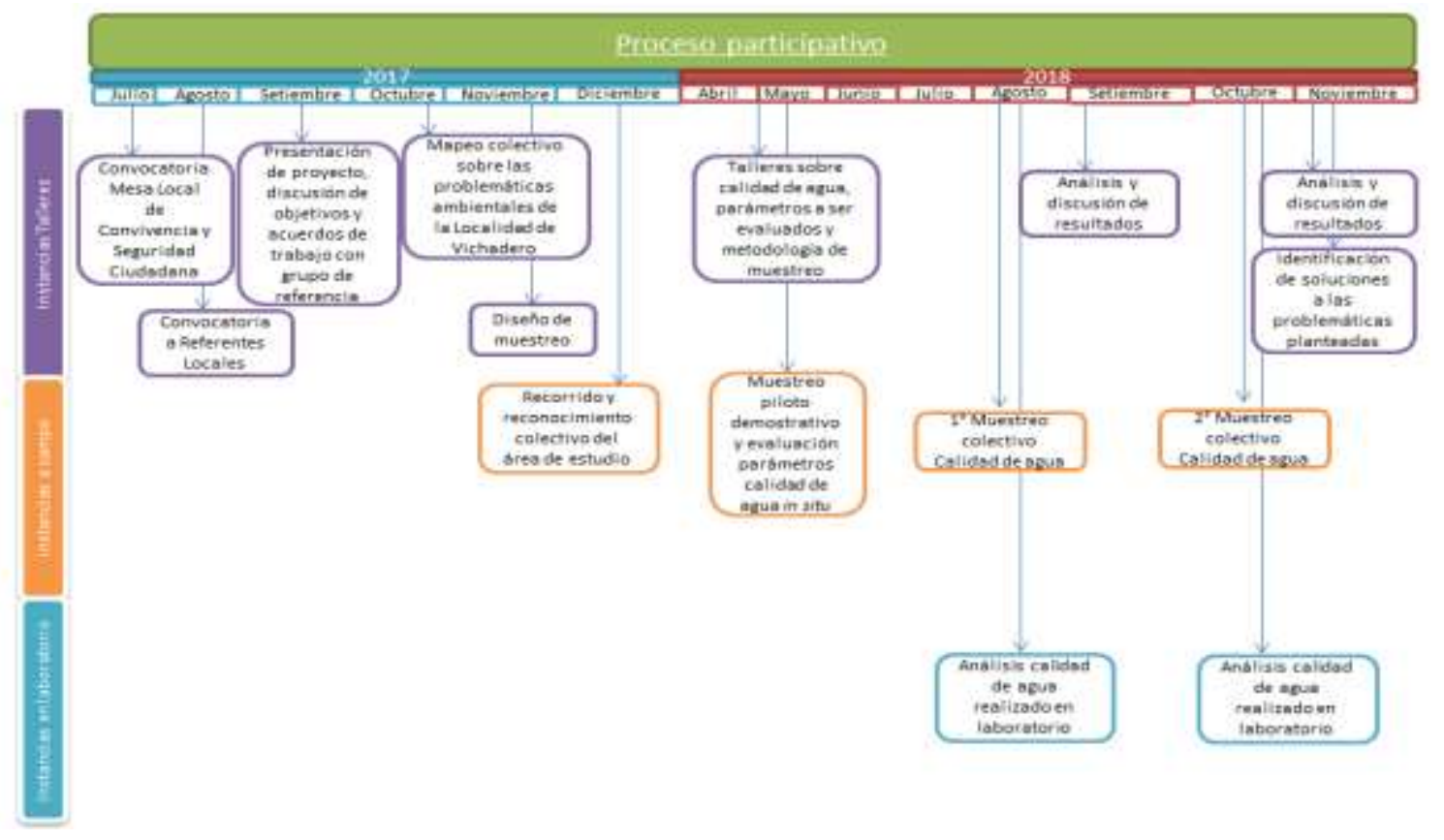

Cuadro 1 Proceso Participativo, línea de tiempo desde julio de 2017 hasta noviembre 2018. 


\section{Convocatoria al grupo de referencia y presentación de proyecto}

En base a que las y los actores sociales poseen saberes específicos, con enfoque en sus conocimientos, prácticas y experiencias locales, en una primera instancia buscando establecer un grupo de referencia se realizó la convocatoria en el municipio de Vichadero dirigida a representantes institucionales de los más diversos ámbitos, organizaciones de la sociedad civil y actores sociales no-universitarios, con fines de formar un grupo de referencia comprometido con los objetivos planteados. La convocatoria al grupo de referencia se realizó en una primera instancia en la Mesa Local para la Convivencia y Seguridad Ciudadana de Vichadero y en segunda instancia se enviaron invitaciones a referentes locales, identificados previamente. En la instancia de convocatoria se realizó una breve presentación de la propuesta, se realizó un intercambio sobre lo planteado y se solicitó a los integrantes de la mesa que extendieran la invitación a participar de la propuesta a posibles interesados.

Una vez formado el grupo de referencia se convocó a una primera reunión de carácter abierto, con el objetivo de presentar la propuesta e intercambiar sobre las problemáticas ambientales en la zona. Se realizó una breve ronda de presentación (nombres, actividad a la que se dedican, si pertenecen a alguna institución y su interés de participar en el grupo) con la finalidad de acercar a las y los distintos actores presentes y poder visualizar el perfil en general del grupo que se estaba por formar. Por parte de las mediadoras (una tesista de grado y una docente del Centro Universitario de Rivera) también se presentó el enmarque de la propuesta presentada, realizando una introducción sobre el Centro Universitario de Rivera.

En cada instancia de encuentro se realizó una introducción a los conceptos generales vinculados a la calidad del agua y a conceptos de carácter técnico sobre monitoreo, parámetros de calidad de agua y sobre la legislación ambiental vigente referida a los cursos de agua e instancias participativas en Uruguay. Se utilizaron recursos y dispositivos múltiples en forma de apoyo y al culminar cada actividad se realizó una puesta en común y una evaluación en conjunto. Para la sistematización de esta experiencia se utilizaron todos los registros escritos, como también grabaciones de voz de reuniones, talleres y actividades de campo. Desde la primera instancia con el grupo de referencia, se trabajó sobre todas las posibilidades de alcance y logros con la propuesta, con la intención de no generar falsas expectativas, generar un buen ambiente de trabajo y lograr el compromiso de las personas involucradas. 


\section{Mapeo Colectivo y diseño del muestreo}

Una vez formado el equipo de referencia se realizó un "mapeo colectivo", con el objetivo de construir las miradas sobre el territorio, identificando los posibles problemas ambientales en la zona y en función de tales problemáticas se ubicaron en el mapa los posibles puntos de muestreo. Se realizó una introducción a la actividad especificando la temática, metodología, tiempo y objetivo. Luego de la presentación se reunieron en tres pequeños grupos bajo la consigna de identificar en el mapa (área de estudio) las posibles problemáticas ambientales. Se utilizaron íconos, recursos visuales e ilustraciones, como elementos disparadores de la discusión y como forma de colaborar en la intervención de las y los participantes. Estos recursos gráficos fueron tomados del Manual de Mapeo Colectivo elaborado por Iconoclasistas, 2013. Al finalizar el ejercicio se realizó una puesta en común en función de los resultados obtenidos, se trabajó sobre un mapa a gran escala para visualizar las problemáticas ambientales en su totalidad y se realizó un posible diseño de muestreo en función de los resultados obtenidos.

Luego de ubicar los posibles puntos de muestreo en el mapa, con intención de profundizar en otras áreas e incorporar dimensiones temporales, corporales y subjetivas, se realizó un recorrido colectivo de reconocimiento del territorio. Se lograron visualizar los posibles conflictos ambientales identificados en el mapeo colectivo, conocer el área de estudio y las actividades productivas de la zona, se hicieron registros fotográficos y se visualizó la accesibilidad que presentan los posibles puntos de muestreo.

En las instancias a campo se realizaron 3 muestreos, el primer muestreo se realizó en un solo punto de los 7 seleccionados. Fue considerado un muestreo piloto y sus resultados no fueron tomados en cuenta en el momento de analizar los datos. Las etapas de muestreo fueron planificadas en forma conjunta, tanto la fecha como el recorrido a ser realizado con el grupo de referencia. Entre cada muestreo se realizaron encuentros entre las y los actores que integran el grupo de referencia para evaluar el proceso hasta el momento, compartir e intercambiar sobre las experiencias con aquellos actores que no pudieron estar presentes en ciertas instancias, plantear dudas y comenzar a analizar los resultados preliminares. Estas instancias de intercambio permitieron unificar, motivar e incentivar al grupo de referencia, 
además de realizar una evaluación constante durante el desarrollo del proceso participativo.

\section{Análisis Calidad de agua y análisis colectivo de resultados}

En la etapa de realización del muestreo colectivo de agua con el grupo de referencia, se determinaron parámetros fisicoquímicos para evaluar la calidad de agua. Los parámetros evaluados fueron los siguientes: temperatura (T), $\mathrm{pH}$, conductividad (K), sólidos disueltos totales (STD), oxígeno disuelto (OD) in situ, contenido de nitratos ( $\mathrm{NO}_{3}{ }^{-}$), fósforo total (PT) y la demanda bioquímica de oxígeno $\left(\mathrm{DBO}_{5}\right)$, comprendidos en la normativa actual para la determinación de la calidad de un curso de agua (Decreto 253/79, 1979)².

Con todos los resultados obtenidos sobre la calidad de agua, se procedió a realizar un análisis integrado, teniendo como prioridad la discusión colectiva y abierta, tratando de integrar los conocimientos adquiridos a lo largo del proceso participativo. Este análisis se detalla a continuación en el ítem discusión de resultados. En esta instancia, también se acordaron en forma colectiva acciones a ser realizadas con los datos obtenidos.

\footnotetext{
${ }^{2} \mathrm{~A}$ su vez aclarar, que no se realizó la determinación de poblaciones particulares de microorganismos como coliformes totales o fecales, parámetros que son tomados en cuenta en el momento de valorar si un curso de agua se puede utilizar con fines recreativos o no, dado que la Intendencia Departamental de Rivera (IDR) realiza análisis microbiológicos de forma rutinaria y dichos datos son de acceso público.
} 


\section{Resultados}

\section{Convocatoria grupo de referencia}

Como resultado de la convocatoria participaron 11 actores locales en el desarrollo del proceso participativo. En el Cuadro 2 se presentan las características de las y los actores participantes.

\begin{tabular}{|c|c|c|c|c|c|c|}
\hline $\begin{array}{c}\mathbf{N}^{\circ} \\
\text { part. }\end{array}$ & Edad & Género & Ocupación & $\begin{array}{c}\text { Participació } \\
\text { n Proceso }\end{array}$ & $\begin{array}{l}\text { Relación } \\
\text { previa con la } \\
\text { temática }\end{array}$ & $\begin{array}{l}\text { Representant } \\
\text { e entidades } \\
\text { gubernament } \\
\text { al }\end{array}$ \\
\hline 1 & $60-70$ & Mujer & $\begin{array}{l}\text { Productora } \\
\text { familiar }\end{array}$ & Alta & SI & NO \\
\hline 2 & $40-50$ & Mujer & Odontóloga & Alta & SI & NO \\
\hline 3 & $50-60$ & Hombre & $\begin{array}{l}\text { Maestro - } \\
\text { Periodista }\end{array}$ & Media & NO & NO \\
\hline 4 & $40-50$ & Mujer & $\begin{array}{l}\text { Veterinaria y } \\
\text { Productora }\end{array}$ & Alta & SI & NO \\
\hline 5 & $40-50$ & Mujer & Escribana & Alta & SI & NO \\
\hline 6 & $50-60$ & Hombre & $\begin{array}{c}\text { Trabajo } \\
\text { informal }\end{array}$ & Media & NO & NO \\
\hline 7 & $50-60$ & Mujer & Maestra & Alta & NO & NO \\
\hline 8 & $60-70$ & Mujer & $\begin{array}{l}\text { Maestra } \\
\text { jubilada }\end{array}$ & Alta & SI & NO \\
\hline 9 & $20-30$ & Mujer & Policía & Baja & NO & SI \\
\hline 10 & $50-60$ & Mujer & Ama de casa & Alta & SI & NO \\
\hline 11 & $40-50$ & Mujer & Médica & Media & SI & SI \\
\hline
\end{tabular}

Cuadro 2 Características de los actores sociales involucrados en el proceso participativo

\section{Diagnóstico participativo y diseño de muestreo a partir de Mapeo Colectivo}

El mapeo colectivo reveló posibles impactos ambientales que pueden incidir en la 
calidad de agua dentro y fuera de la planta urbana del municipio de Vichadero. Las problemáticas ambientales identificadas fueron clasificadas en función de las microcuencas.

El grupo de referencia no identificó problemas ambientales puntuales dentro de la microcuenca del arroyo Ceibal, por lo cual la misma no fue incluida en el diseño de muestreo.

Problemáticas ambientales en la microcuenca del arroyo Coronilla Chico:

- Uso de agroquímicos y calidad de agua: el grupo de referencia manifiesta en forma general su preocupación por el uso de agroquímicos en la zona y su presencia en los cuerpos de aguas superficiales de la microcuenca en cuestión y en particular hace referencia al sistema de suministro de agua que se extrae del arroyo Coronilla Chico. En este arroyo se realizó un represamiento, con la finalidad de almacenar agua suficiente para el consumo diario de la población. Sobre la microcuenca del arroyo Coronilla Chico, aguas abajo, se encuentra el balneario "Ceibal", utilizado como espacio de recreación y baño por las y los habitantes del municipio y sus alrededores. El grupo de referencia también manifestó preocupación sobre la calidad de agua del balneario.

\section{Problemáticas ambientales en la microcuenca del arroyo Coronilla}

-Ausencia de red generalizada de Saneamiento: actualmente Vichadero no cuenta con una red de saneamiento generalizada, el mayor porcentaje de los vertidos de aguas servidas escurren en forma directa a los cursos de agua presentes en la planta urbana.

-Gestión de residuos: el grupo de referencia manifiesta la ausencia de manejo de residuos como una problemática ambiental del municipio, a pesar que existe recolección domiciliaria y contenedores en las principales vías públicas. A su vez la mayor preocupación manifestada hace referencia al vertedero municipal (trinchera sanitaria), destino final de los residuos. El vertedero está ubicado en la zona alta de la microcuenca del arroyo Coronilla, las y los actores locales también manifiestan preocupación respecto al manejo de residuos fitosanitarios que también tienen como destino final los cursos de agua de la zona.

-Impactos vinculados a la producción rural: la existencia de silos y barracas de cuero en las zonas marginales del municipio también es manifestado como una preocupación del grupo de referencia. Otra problemática manifestada es la existencia de barracas de tratamiento de cuero que generan olores desagradables cuyas aguas residuales son vertidas al arroyo Coronilla. También son consideradas una problemática ambiental el uso y especialmente la falta de fiscalización del uso adecuado de agroquímicos. 
En función de estas problemáticas ambientales anteriormente identificadas y seleccionadas como prioritarias junto al grupo de referencia, fueron definidos los 7 puntos de muestreo (Cuadro 3). Tres puntos se localizaron sobre la microcuenca alta del arroyo Coronilla, incluyendo el punto control, ubicado aguas arriba del arroyo Coronilla, dentro de un predio privado con certificado de ganadería orgánica (ubicado a dos kilómetros aproximadamente de la represa de OSE) y cuatro puntos fueron ubicados sobre la microcuenca alta del arroyo Coronilla. En los talleres previos a los muestreos se acordaron pautas comunes de muestreo, utilizando métodos sencillos, alcanzables por todas y todos y con compromiso real de las partes involucradas (cómo registrar los datos y colectar las muestras).

\begin{tabular}{|llll|l|}
\hline Puntos & Microcuenca & Referencia & Coordenada_ X & Coordenada_y \\
\hline 0 & $A^{\circ}$ Coronilla Chico & Punto Control & 717918,7 & 6484973,6 \\
\hline 1 & $A^{\circ}$ Coronilla Chico & Represa & 718458,4 & 6485061 \\
\hline 2 & $A^{\circ}$ Coronilla Chico & Ceibal & 722618,1 & 6484561,8 \\
\hline 3 & $A^{\circ}$ Coronilla & Mevir 2 & 718050,9 & 6482290,5 \\
\hline 4 & $A^{\circ}$ Coronilla & Mevir 1 & 717715,8 & 6481465,3 \\
\hline 5 & $A^{\circ}$ Coronilla & Vertedero & 716111,7 & 6480594,5 \\
\hline 6 & $A^{\circ}$ Coronilla & Paso del Parque & 714450,1 & 6478325,5 \\
\hline
\end{tabular}

Cuadro 3 Puntos de Muestreo

\section{Parámetros Calidad de agua}

Se realizaron dos muestreos en conjunto con el grupo de referencia, el primer muestreo realizado el 2/08/2018, según información solicitada al Instituto Uruguayo de Meteorología (INUMET), presentó una precipitación acumulada en las 72 horas anteriores al muestreo de $13 \mathrm{~mm}$ y temperatura media de $12^{\circ} \mathrm{C}$ aproximadamente (INUMET). El segundo muestreo, realizado el 30/10/2018, presentó una precipitación acumulada en las 72 horas 
anteriores al $25^{\circ}$ muestreo de $1,5 \mathrm{~mm}$ (INUMET) y una temperatura media de $25^{\circ} \mathrm{C}$ (INUMET). Ambos muestreos se realizaron en días con cielo despejado.

En términos generales, en ambos muestreos los parámetros evaluados in situ de la calidad de agua, como ser T, pH, K, STD, OD; presentaron los valores más bajos en el punto de muestreo seleccionado como punto de control (punto 0), ubicado sobre la microcuenca del arroyo Coronilla Chico, con excepción del OD. Los valores máximos en ambos muestreos de K y STD corresponden al punto de muestreo cercano a la pileta de saneamiento de MEVIR 1 (punto 4), ubicado sobre la microcuenca del arroyo Coronilla ( Figura 3).
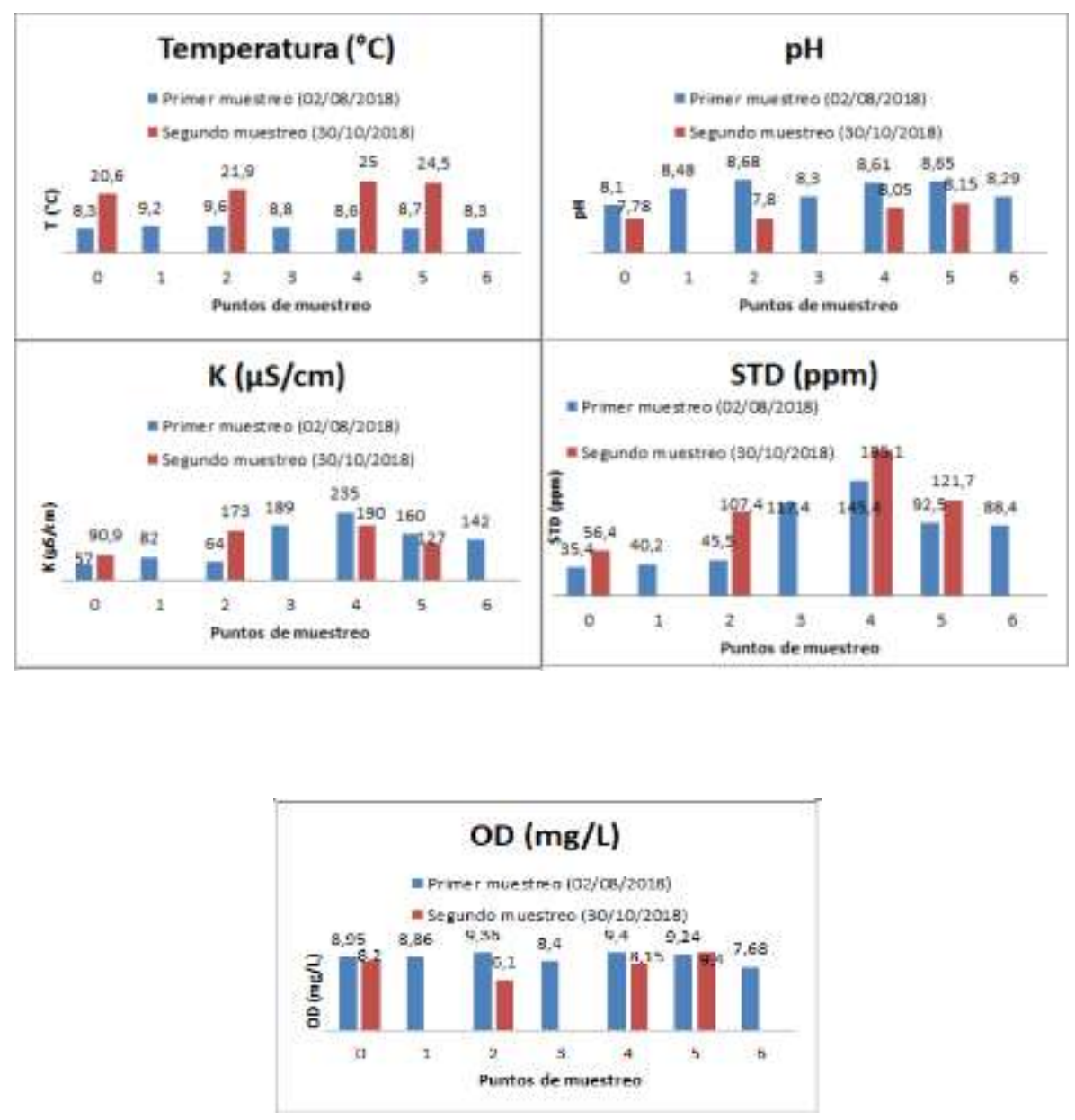

Figura 3. Parámetros físico-químicos de la calidad de agua, evaluados in situ.

La figura 4 abajo, nos permite visualizar por microcuenca los valores obtenidos de los parámetros físico-químicos evaluados en laboratorio. Sobre la microcuenca del arroyo 
Coronilla Chico los valores máximos de $\mathrm{NO}_{3}$, $\mathrm{PT}$ y $\mathrm{DBO}_{5}$ alcanzados corresponden al punto 2 (Balneario Ceibal) y los valores mínimos en el punto 0, tomado como punto control. Sobre la microcuenca del arroyo Coronilla se observa que los mínimos valores corresponden al punto 6 (Paso del Parque) y los máximos valores respecto al $\mathrm{NO}_{3}^{-}$y al $\mathrm{DBO}_{5}$ al punto 4 cercano a pileta saneamiento MEVIR 1 y el PT máximo corresponde al punto 5 próximo al vertedero municipal.

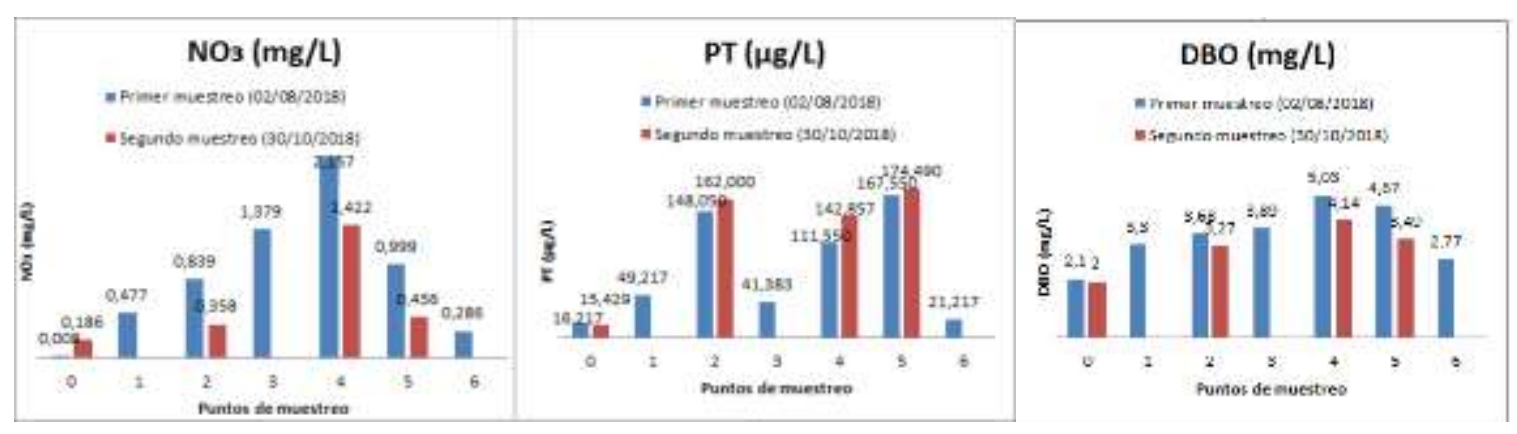

Figura 4. Parámetros físico-químicos de la calidad de agua, evaluados en laboratorio.

Todos los valores de los parámetros de calidad de agua evaluados tanto in-situ como en laboratorio se encuentran dentro de las referencias establecidas por decreto reglamentario 253/979 que hace referencia a la normativa en materia de calidad de agua en Uruguay, en excepción del PT que se encuentra por encima de los límites establecidos ( $25 \mu \mathrm{g} / \mathrm{L}$ ) en 5 de los puntos muestreados (ver Figura 4), salvo el punto 0 (punto control) y el punto 6, Paso del Parque.

\section{Discusión}

En este ítem se procederá a discutir y comparar la percepción y el grado de conocimiento que el grupo de referencia tiene sobre la calidad de agua de su entorno, con los resultados obtenidos respecto a los parámetros de calidad de agua evaluados en las microcuencas del arroyo Coronilla y arroyo Coronilla Chico.

Apuntando al grado de participación y en cuanto a la relación previa con la temática, 6 de las 7 personas que afirmaron tener relación previa con la temática, estas participaron en forma activa en el grupo de referencia (conforme al Cuadro 2), con un alto grado de motivación, tanto en la asistencia como en la participación, buscando involucrarse y 
comprender todas las etapas del proceso de obtención de datos sobre calidad de agua. La integrante de número 11 afirma tener una relación previa con la temática, pero por motivos laborales no tuvo una incidencia mayor en el proceso. Por otro lado, involucrar a representantes institucionales gubernamentales locales no ha garantizado una participación activa.

Una de las manifestaciones sobre la asociación entre la participación activa y el conocimiento previo de la temática, fue la preocupación entre las problemáticas ambientales identificadas y su influencia en la salud de la comunidad. Otra de las manifestaciones sobre la asociación entre la participación activa y el conocimiento previo de la temática fue manifestada por las participantes 1 y 2, quienes indicaron sus preocupaciones con el uso de agroquímicos que se está llevando a cabo en la zona, principalmente en las formas de dispersión y en la ausencia de control de su uso en las cercanías de la represa de OSE, ubicado en la microcuenca arroyo Coronilla Chico. En estos casos la relación previa con la temática se establece en función de sus ocupaciones laborales, por sus vínculos cercanos con el territorio, las afectaciones en forma directa y por su actual participación en movimientos sociales ambientales.

En esta misma línea Abreu et al., (2017) afirma que:

"la percepción de actores locales en sus relaciones de trabajo y moradia, cuando es llevada en consideración en estudios de diagnóstico socio-ambiental, puede esclarecer flujos y dinámicas sociales de vida de los territorios para la apropiación efectiva de situaciones problemáticas vivenciadas y contribuir para el fortalecimiento comunitario en defensa de condiciones ambientales de menor riesgo a la salud (...)” (2015, p. 3804).

El comportamiento de los parámetros de calidad de agua en las dos microcuencas abordadas es similar en los dos muestreos, encontrándose una tendencia a que los valores máximos y mínimos observados de cada parámetro se asocian a determinados puntos de muestreo. Las diferencias mínimas observadas se podrían atribuir a las condiciones climáticas 
correspondientes de cada muestreo.

Por otra parte, observamos que el punto de muestreo seleccionado como punto control (ubicado dentro de un predio privado con certificado ecológico) presenta los menores valores de todos los parámetros de calidad de agua evaluados, indicando que la elección de este punto control fue adecuada. Los niveles observados de PT, indican que es necesario profundizar en la hipótesis de que los distintos usos de suelos generan un impacto diferente en la calidad de agua de los recursos hídricos, dentro de las microcuencas estudiadas (Matson, Parton, Power y Swift, 1997). De esta forma son compatibles con la definición de los puntos de muestreo que fueron seleccionados en base a los usos de suelos que presentan problemáticas ambientales.

En cuanto a las problemáticas ambientales identificadas a lo largo del proceso participativo en base a la percepción del grupo de referencia, se procede a realizar una correlación con los parámetros de calidad de agua superficiales.

Dentro de la microcuenca del arroyo Coronilla Chico, el PT presentó un incremento muy marcado entre el punto de control y los puntos 2 y 3, el cual superó ampliamente el máximo establecido por el decreto 253/979 (25 $\mu \mathrm{g} / \mathrm{L})$, esto ocurrió tanto en el primer como en el segundo muestreo. Cabe destacar que la distancia aproximada entre el punto de control y el punto 1 es de aproximadamente un kilómetro. Probablemente este incremento está asociado a la escorrentía superficial, a la desmineralización de los suelos y a los usos de suelos relacionados a actividades agrícolas de la zona, tres fuentes de pérdida de fósforo sobre todo en aguas superficiales. Este incremento se observa principalmente en el primer muestreo, donde hubo mayores precipitaciones acumuladas a las 72 horas anteriores al muestreo, ya que estas vía está limitada espacialmente y temporalmente a eventos de lluvia de alta magnitud e intensidad (Heathwaite, 2000).

En la microcuenca del arroyo Coronilla los parámetros evaluados en los diferentes puntos no presentan el mismo comportamiento que los puntos de muestreo ubicados en la microcuenca estudiada anteriormente, teniendo en cuenta que el arroyo Coronilla tiene sus nacientes en la planta urbana del municipio, incidiendo la misma en sus condiciones. En base 
a estas características que presentan ambas microcuencas se da evidencia que para interpretar la relación entre las actividades presentes en un territorio y la calidad de agua se debe tomar como unidad espacial de análisis a las cuencas hidrográficas (Achkar et al., 2016).

Con respecto a las preocupaciones manifestadas con relación a la contaminación de los cuerpos de agua y su vínculo con el uso de agroquímicos, se ha discutido y consensuado la insuficiencia de muestreos realizados para afirmar tales suposiciones, para eso se necesita realizar un monitoreo continuo y sistemático. En función a eso el grupo de referencia ha planteado impulsar la creación de un observatorio socio-ambiental en la región y se hizo hincapié en la importancia de divulgar en forma conjunta los resultados obtenidos en este trabajo en las diferentes instituciones educativas y de salud.

\section{Conclusiones}

Esta investigación nos permitió identificar algunas de las principales problemáticas ambientales de la zona de estudio, a partir de un diagnóstico socio-ambiental realizado en conjunto con el grupo de referencia, utilizando metodologías que promueven la participación de actores sociales locales. Además de presentar una aproximación a una línea de base sobre el comportamiento de ciertos parámetros de calidad de agua, lo que se desprende es la necesidad de realizar un monitoreo continuo y sistemático de los cuerpos de agua de la zona, con la finalidad de lograr un manejo integrado de cuenca a nivel local.

Con respecto a los objetivos planteados, esta investigación nos permite afirmar que existe una relación directa entre la percepción que tienen las y los actores sociales locales que integraron el grupo de referencia sobre la calidad de agua, en relación a los parámetros de calidad de agua evaluados en las microcuencas de los arroyo Coronilla y arroyo Coronilla Chico. Por esta razón entendemos que es importante incorporar la percepción de actores locales sobre su entorno en espacios académicos, así como también en formulación de políticas públicas, una vez que son las y los principales afectados por las mismas.

Las microcuencas estudiadas presentaron comportamientos diferentes y complejos respecto a la presencia de aportes puntuales y difusos. En términos generales los parámetros evaluados se encuentran dentro de los límites establecidos por la legislación, a excepción del 
fósforo total. A nivel nacional según el Plan Nacional de Aguas (MVOTMA, 2017) en todas las cuencas monitoreadas se excede holgadamente los límites establecidos en este nutriente.

Este tipo de investigación con estrategia participativa, que tiene como uno de sus objetivos abordar la percepción de actores sociales locales sobre problemáticas ambientales, dan evidencia que procesos con similares características podrían promover el involucramiento de actores locales en procesos reales de gestión ambiental de su comunidad.

A su vez este diagnóstico socio-ambiental permitió prever acciones a corto y largo plazo respecto a las problemáticas ambientales que se identificaron. A corto plazo se trató de abordar aquellas problemáticas referidas al cumplimiento de la legislación vigente y de realizar difusión de los resultados en instituciones educativas. A largo plazo se planteó la necesidad de implementar un observatorio socio-ambiental participativo en la localidad, con el objetivo de realizar un monitoreo real continuo y sistemático de la problemáticas ambientales ya identificadas en este trabajo.

\section{Referencias}

Abreu, M., Sabóia, V., Nascimento, C. y de Souza, S. (2017). Diagnóstico participativo socioambiental e de riscos à saúde das comunidades do entorno do Complexo Petroquímico do Rio de Janeiro, Brasil. Ciência Saúde Coletiva, 22 (11), 3793-3805.

Achkar, M., Domínguez, A. y Pesce, F. (2004). Diagnóstico Socioambiental Participativo en Uruguay. Montevideo, Uruguay: Redes AT.

Achkar, M., Díaz, I., Domínguez, A. y Pesce, F. (2016).Uruguay: Naturaleza, sociedad, economía. Una visión desde la Geografía. Montevideo, Uruguay: Banda Oriental.

Aguirre, M. (2011). La cuenca hidrográfica en la gestión integrada de los recursos hídricos. Revista Virtual REDESMA, 5 (1), 9-20.

Balcazar, F.E. (2003). Investigación acción participativa (IAP): Aspectos conceptuales y dificultades de implementación. Fundamentos en humanidades, Universidad Nacional de San 
Luís, 4 (7), 59-77.

Benez, M.C., Kauffer Michel, E.F. y Álvarez Gordillo, G.C. (2010). Percepciones Ambientales de la Calidad del Agua Superficial en la microcuenca del Río Fogótico, Chiapas. México. Frontera Norte, 22 (43), 129-158.

Brandao, G.C. (2014). Development of a simple method for the determination of nitrite and nitrate in ground water by high-resolution continuum source electro thermal molecular absorption spectrometry. Analytica Chimica Acta, 2 (806), 101-106.

Chaui, M. (1996). Convite à filosofia. São Paulo, Brasil: Ática.

De León L. (2011). Evaluación de la calidad del agua del río Cuareim, período 2006- 2010. Proyecto DINAMA-PNUD. RU/07/012-TDR 3.12.

Dirección Nacional de Medio Ambiente [DINAMA] (2009a). Manual de procedimientos analíticos para muestras ambientales 2008UY: Determinación de Demanda Bioquímica de Oxígeno en efluentes líquidos domésticos e industriales, aguas contaminadas y naturales. Método electrométrico.

Dirección Nacional de Medio Ambiente [DINAMA] (2009b). Manual de procedimientos analíticos para muestras ambientales 4013UY: Determinación de Fósforo total en aguas naturales y tratadas, aguas residuales domésticas e industriales. Método Espectrofotométrico.

Dirección Nacional de Ordenamiento Territorial [DINOT]. (2015). Cobertura de categorización de usos del suelo. Recuperado de: http://sit.mvotma.gub.uy/shapefiles.htm.

Environmental Systems Research Institute [ESRI]. (2012). ArcGis 10.1. ArcMap Versión 10.1. License Type ArcInfo. Copyright (C) 1999-2012 ESRI Inc.

Eschenhagen, M. L. (2007). La educación ambiental superior en América Latina: una evaluación de la oferta de posgrados ambientales. Theomai, 16 (16), 87-107. 
Fals Borda, O. (1981). La ciencia y el pueblo, en Investigación Participativa y Praxis Rural. Lima, Perú: Mosca Azul.

Fernández, G. (2005). Los sistemas de información geográfica (SIG) como herramienta para el ordenamiento ambiental del territorio. En: Achkar, M.; Cantón, V.; Cayssials, R.; Domínguez, A.; Fernández, G. y Pesce, F. (Edición DIRAC), Ordenamiento Ambiental del Territorio (pp.93-102). Facultad de Ciencias. Montevideo, Uruguay: UdelaR CSEP.

Fernández, Y. (2008). ¿Por qué estudiar las percepciones ambientales?: Una revisión de la literatura mexicana con énfasis en Áreas Naturales Protegidas. Espiral, (Guadalajara), 15 (43), 179-202.

Geilfus, F. (2002). 80 herramientas para el desarrollo participativo. Diagnóstico, planificación, monitoreo y evaluación. San José, Costa Rica: IICA.

Grosse, R., Santos, C., Taks, J. y Thimel, S. (compiladores) (2006). Las canillas abiertas de América Latina II. La lucha contra la privatización del agua y los desafíos de una gestión participativa y sustentable de los recursos hídricos. Montevideo, Uruguay: Casa Bertolt Brecht.

Heathwaite, A.L.(2000). Character is in phosphorusloss in surface and sub surface hydrological path ways. Science of the total environment, 38 (523), 251-252.

Instituto de Hidrología, Meteorología y Estudios Ambientales [IDEAM]. (2007) Nitrato en agua por espectrofotometría ultravioleta. Código: TP0092.

Instituto Nacional de Estadística [INE]. (2011). Censo de Población 2011, Rivera.

Instituto Uruguayo de Meteorología [INUMET]. (2018). Datos no publicados.

Ley Nacional N 14.859. Diario Oficial de la República Oriental del Uruguay. Montevideo, 
Uruguay, 15 de diciembre de 1978.

Ley Nacional N 18.610. Diario Oficial de la República Oriental del Uruguay. Montevideo, Uruguay, 28 de octubre de 2009.

Matson, P., Parton, W., Power, A. y Swift, M. (1997). Agricultural intensification and Ecosystem properties. Science, 277 (5325), 504-509.

Ministerio de Vivienda Ordenamiento Territorial y Medio Ambiente [MVOTMA]. (2017). Plan Nacional de Aguas. Montevideo, Uruguay.

Ordenanza Ordenamiento Territorial del Departamento de Rivera № 03/2007, Intendencia Departamental de Rivera, Uruguay, versión 20 de diciembre de 2018. Recuperado de: www.rivera.gub.uy

Programa de las Naciones Unidas para el medio ambiente [PNUMA]. (2009). Perspectivas del Medio Ambiente Urbano: GEO Localidades urbanas de Rivera, Minas de Corrales, Tranqueras y Vichadero. Montevideo, Uruguay: Ceuta.

Ríos, M. (2012). Evaluación participativa de impactos de los plaguicidas utilizados en soja y forestación en un área protegida y su cuenca (Programa de Maestría de Ciencias Ambientales). Facultad de Ciencias - Universidad de la República, Uruguay.

Risler, J. y Ares, P. (2013). ICONOCLASISTAS Manual de mapeo colectivo: recursos cartográficos críticos para procesos de creación colaborativa. Buenos Aires, Argentina: Tinta Limón.

Sevilla Guzmán, E. (2001) Agroecología y desarrollo rural sustentable: una propuesta desde Latinoamérica. En: Sarandon, S. (Ed.), Agroecología. El camino hacia una agricultura sustentable (25). Buenos Aires, Argentina: Ediciones Científicas Americanas. Vargas, L. M. (1994). Sobre el concepto de percepción. Alteridades, 4 (8), 47-53. 